Zbl Arbeitsmed 2015 • 65:190-191

DOI 10.1007/s40664-015-0029-9

Online publiziert: 18. Juni 2015

(c) Springer-Verlag Berlin Heidelberg 2015

\section{D.A. Groneberg}

Institut für Arbeitsmedizin, Sozialmedizin und Umweltmedizin, Goethe-

Universität Frankfurt am Main, Frankfurt am Main, Deutschland

\title{
Betrachtung der Additivität von Exzessrisiken bei verschiedenen Expositionskonstellationen
}

Der in dieser Ausgabe des Zentralblatts von Morfeld und Spallek publizierte Beitrag mit dem Titel „Gefährdungsbeurteilung bei Exposition gegenüber mehreren krebserzeugenden Arbeitsstoffen “ greift eine aktuelle und wichtige Thematik auf, die zum Teil kontrovers diskutiert wird [1].

Bei der Lektüre der Arbeit ist das strikte Unterscheiden von Begrifflichkeiten wichtig, um Missverständnisse zu vermeiden: Man darf den Autoren keinesfalls unterstellen, dass sie nur die Additivität von Expositionen untersuchen, also z. B. der Frage nachgehen, ob beispielsweise $\mathrm{PAH}$ und Asbestexpositionen addiert werden können. Zur Klarstellung: Morfeld und Spallek betonen, dass sie „ausschließlich die Additivität von Exzessrisiken bei verschiedenen Expositionskonstellationen untersuchen “. Sie führen aus, dass Exzessrisiken unabhängig von der einwirkenden Exposition stets dieselbe Maßeinheit haben und man daher sinnvoll nach Ordnung und Additivität dieser Exzessrisiken fragen kann. Eine Addition von Expositionen, die auf verschiedenen Skalen mit unterschiedlichen Maßeinheiten definiert sind, mache im Gegensatz dazu keinen Sinn [z. B. PAH (polyzyklische aromatische Kohlenwasserstoffe)-Exposition gemessen in $\mu \mathrm{g} / \mathrm{m}^{3}$-Jahre, aber Asbestexposition in Faserjahren] und ist nicht Gegenstand der vorliegenden Arbeit.

$\mathrm{Zu}$ der Aktualität der Thematik findet sich seitens der Deutschen Gesetzlichen Unfallversicherung (DGUV) folgendes Statement im Bereich „Fragen aus der Praxis - Antworten der DGUV“: Frage: Sind bei einer gleichzeitigen Exposition gegenüber mehreren Stoffen mit risikobezogenen Beurteilungsmaßstäben nach
TRGS 910 additive oder überadditive (synergistische) Effekte zu erwarten und ist deshalb grundsätzlich ein Bewertungsindex nach TRGS 402 zu bilden? Antwort der DGUV: Der AGS erarbeitet ein Konzept zur Bewertung von Kombinationswirkungen mehrerer krebserzeugender Stoffe. Bis dahin legt die TRGS 402 (2014) fest: Sofern eine Exposition gegenüber mehreren Kanzerogenen vorliegt, werden diese als Einzelstoffe bewertet. Ein Bewertungsindex ist nicht zu berechnen [2].

In dem vorliegenden, theoretischen Beitrag beziehen sich Morfeld und Spallek bezüglich ihrer Eingangshypothesen auf die Vorstellungen Dritter, dass einfache Summationsformeln von Einzelrisiken geeignet sind und entsprechende Vorteile bieten bei einer Gefährdungsbeurteilung gegenüber mehreren krebserzeugenden Arbeitsstoffen gegenüber einer Einzelrisikobetrachtung. Aufbauend auf diesen Hypothesen werden mithilfe mathematischer Modelle Erklärungen gesucht.

Auch wenn man die Eingangsannahmen ablehnen sollte - was jedoch wissenschaftlich begründet werden muss - so kann man nicht grundsätzlich die abgeleiteten Ergebnisse a priori ablehnen, da falschen Annahmen nicht notwendig falsche Aussagen folgen. Seit dem Mittelalter erklärt dies die Aussagenlogik „ex falso sequitur quodlibet et verum sequitur ex quolibet“ (Petrus Hispanus, Summae Logicales, verfasst um 1240).

Die Autoren gehen auf Limitationen ihrer Hypothesen ein und benennen diese: So berücksichtigt ihr Ansatz die Variablen nicht stetig, und der individuelle Response ist nicht probabilistisch formuliert.

Außerdem machen sie deutlich, dass sich Kausalwirkungen nicht notwendig in einer Risikoänderung, also in Zusatzfällen, sondern auch in einer zeitlichen Verlagerung des Ereigniseintritts äußern können. Sie schlussfolgern, dass ihr Ansatz all dieses nicht abdeckt und entsprechend $\mathrm{zu}$ erweitern ist. Allerdings betreffen diese Punkte nicht die aktuellen in Deutschland bestehenden oder vorgeschlagenen Konzepte, auf die Autoren in ihrer Arbeit Bezug nehmen und sich in ihrer Argumentation beschränken.

Deswegen ist es auch wichtig festzuhalten, dass sich Morfeld und Spallek vor allem mit ihrer Zielrichtung auf den Ausschuss für Gefahrstoffe (AGS), also Prävention und Grenzwertsetzung beziehen und weniger auf das Berufskrankheitenrecht.

\section{> Die Teilkausalität ist bei \\ Berufskrankheiten bei einem \\ Exzessrisiko $>50 \%$ gegeben.}

Deshalb ist die Frage aufgekommen, ob 2 Expositionen gemeinsam das kritische Exzessrisiko überschreiten können, während für jede der beiden Einzelexpositionen die Teilkausalität nicht gegeben ist. Hier geht es also um das Zusammenwirken zweier Expositionen auf einen medizinischen Endpunkt, und dies ist auch im BK-Recht real umgesetzt, denn es gibt eine Berufskrankheiten-Situation „Lungenkrebs nach Asbest und PAKs“, bei der es zentral um die Synergie der beiden Expositionen geht. Hier hat der Beitrag zum Exzessrisiko nach Doppelexposition also auch eine gewisse Relevanz, allerdings nur in diesem Fall. Und nur diese besondere BK-Situation zitieren und besprechen die Autoren in ihrer Arbeit. 
Es bleibt abzuwarten, wie der vorliegende Beitrag von den mit der Thematik befassten Experten aufgenommen wird. Seiner Tradition folgend, wird das Zentralblatt auch in diesem Fall gerne zielgerichtete und fundierte Stellungnahmen dazu publizieren und den Autoren Morfeld und Spallek zur Beantwortung vorlegen.

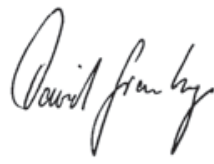

Prof. Dr. D.A. Groneberg

Editor-in-Chief

\section{Korrespondenzadresse}

\section{Prof. Dr. D.A. Groneberg}

Institut für Arbeitsmedizin, Sozialmedizin und Umweltmedizin

Goethe-Universität Frankfurt am Main

Theodor-Stern-Kai 7, 60590

Frankfurt am Main

arbsozmed@uni-frankfurt.de

\section{Einhaltung ethischer Richtlinien}

Interessenkonflikt. D.A. Groneberg gibt an, dass kein Interessenkonflikt besteht.

\section{Literatur}

1. Morfeld B, Spallek M (2015) Gefährdungsbeurteilung bei Exposition gegenüber mehreren krebserzeugenden Arbeitsstoffen. Zentralbl Arbeitsmed Arbeitsschutz Ergonomie 65. DOI:10.1007/s40664015-0032-1.

2. http://www.dguv.de/ifa/Fachinfos/Exposition-Risiko-Beziehung-(ERB)/Fragen-aus-der-Praxis-Antworten-der-DGUV/Allgemeine-Fragen/index.jsp

\section{Traumatische Gelenkinstabilitäten der oberen Extremität}

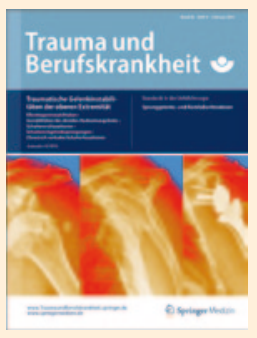

Bereits geringe Einschränkungen an Hand-, Ellenbogenund Schultergelenk können zu großen funktionellen Defiziten führen. Als Folge resultieren oft lange, chronische

Heilungsverläufe und Minderung der Erwerbsfähigkeit. Durch neue Erkenntnisse ergeben sich immer differenziertere und teilweise auch kontrovers diskutierte Therapieoptionen.

Die Zeitschrift Trauma und Berufskrankheit greift in der Ausgabe 4/2014 die im Fokus stehenden Gelenkpathologien auf und verschafft Ihnen einen kompakten Überblick zu Therapiemöglichkeiten und zu erwartenden Behandlungsergebnissen.

Lesen Sie im Schwerpunktheft mehr zu folgenden Themen:

- Ligamentäre Stabilisierungsverfahren bei chronischer Ellenbogeninstabilität

- Knochenaufbauplastiken am Processus coronoideus ulnae

- Essex-Lopresti-Verletzungen

- Das chronisch dezentrierte und instabile distale Radioulnargelenk

- Traumatische anteriore Schultererstluxation

- Schultereckgelenksprengungen

- Behandlungsmöglichkeiten der chronisch verhakten Schulterluxation

Bestellen Sie diese Ausgabe zum Preis von

47,- EUR zzgl. Versandkosten bei

Springer Customer Service Center

Kundenservice Zeitschriften

Haberstr. 7, 69126 Heidelberg

Tel.: +49 6221-345-4303

Fax: +49 6221-345-4229

E-Mail: leserservice@springer.com

Suchen Sie noch mehr zum Thema? Mit e.Med, dem Online-Paket von Springer Medizin, können Sie schnell und komfortabel in über 600 medizinischen Fachzeitschriften recherchieren.

Weitere Infos unter springermedizin.de/eMed.

\section{Keine Geschäfte mit Organspenden}

Die Deutsche Gesellschaft für Orthopädie und Unfallchirurgie e.V. (DGOU) fordert vom Bundesministerium der Finanzen (BMF) die Korrektur des Umsatzsteueranwendungserlasses, der seit 1.1.2015 menschliche Knochenspenden nicht mehr als Organspenden ansieht. Die neue Auslegung des Organbegriffes durch das BMF führt zukünftig zur Besteuerung von allogenen menschlichen Knochen.

Das Umsatzsteuergesetz sieht im Paragraph $4 \mathrm{Nr} .17$ a vor, menschliche Organe, menschliches Blut und Frauenmilch von der Umsatzsteuer zu befreien. Mit der neuen Verwaltungsauffassung zum Organbegriff greift das BMF in die bisher in Deutschland seit Jahrzehnten geltenden Befreiungsvorschriften für die Aufbereitung und Bereitstellung menschlicher Organ- und Gewebeteile ein. Bisher war ohne jegliche Unterscheidung zwischen einzelnen Gewebearten die Lieferung menschlicher Gewebetransplantate zur therapeutischen Anwendung ausnahmslos umsatzsteuerbefreit. Nun lässt sich die Änderung nicht mehr mit den ethischen und gesundheitspolitischen Basisprinzipien der Europäischen Union vereinbaren, laut derer ein Verbot besteht, den menschlichen Körper und Teile davon zur Erzielung von Gewinnen zu nutzen.

Die DGOU hält eine staatliche Einkommenserzielung in Form einer Umsatzsteuer auf Knochenspenden und -transplantate für falsch. Zudem würden die Konsequenzen dieses Umsatzsteueranwendungserlasses die Versorgung von Patienten mit medizinisch notwendigen Knochentransplantaten in Deutschland möglicherweise nachhaltig beeinträchtigen.

Quelle: Deutsche Gesellschaft für Orthopädie und Unfallchirurgie e. V., www.dgou.de 